\title{
Liaisons entre efforts de coupe, densité et états de surface de quelques composites à base de bois
}

\author{
A. ZERIZER ${ }^{1, a}$ et P. MARTiN ${ }^{2}$ \\ 1 Faculté des sciences de l'ingénieur, L.M.M.C., Université M'hamed Bougara, 35000 Boumerdes, Algérie \\ 2 École Nationale Supérieure d'Arts et Métiers, 4 rue Augustin Fresnel, 57078 Metz, France
}

Reçu le 9 juin 2005, accepté le 14 novembre 2006

\begin{abstract}
Résumé - Divers échantillons de composites à base de bois ont été étudiés du point de vue de leur comportement à l'usinage, de la qualité des surfaces produites et de leurs caractéristiques densitométriques. On a mis en évidence, l'influence de la densité et des conditions de coupe à travers $E_{\mathrm{m}}$ (épaisseur moyenne du copeau) de ces panneaux sur les puissances et efforts de coupe, mais aussi la liaison de ces efforts avec la variation de la rugosité de surface de chants usinés, représentée par l'écart moyen $R_{\mathrm{a}}$ qui tient compte de la moyenne arithmétique des rugosités détectées le long d'un profil mesuré.
\end{abstract}

Mots clés : Usinage / composites bois / efforts de coupe

Abstract - Relationships between cutting forces, density and surface roughness of some wood panels. Various samples of wood pannels have been studied from a viewpoint of woodcutting, surface quality produced and of their density characteristics. We have shown the effect of density and cutting conditions through $E_{\mathrm{m}}$ (average chip thickness) of these panels on the power and cutting forces required, but also the correlation linking these cutting forces with the variation of quality (roughness) of knife planed surface, represented by the mean arithmetical deviation $R_{\mathrm{a}}$ that takes account of the arithmetic average of the roughness detected along a measured profile.

Key words: Woodcutting / wood panels / cutting forces

\section{Introduction}

Une grande partie du bois consommé dans le monde est utilisée après déstructuration et reconstitution sous forme de composites : panneaux de particules, panneaux de fibres, LVL (Laminated Veneer Lumber), MDF (Medium Density Fiberboard), OSB (Oriented Strand Boards)... L'existence de ces produits permet de bien mettre en valeur les qualités et performances du bois (Fig. 1).

L'association du bois et d'autres matériaux est l'un des enjeux forts de l'innovation technico-économique dans le domaine des composites à base de bois :

- Prise en charge d'impératifs techniques ayant pour objectif de conserver les caractéristiques avantageuses du bois massif (performances mécaniques spécifiques, performances thermiques (isolation) et aspect) ; tout en atténuant les inconvénients du bois massif (variations dimensionnelles, altérabilité biologique et variabilité).

\footnotetext{
a Auteur correspondant : zerizer_ab@yahoo.fr
}

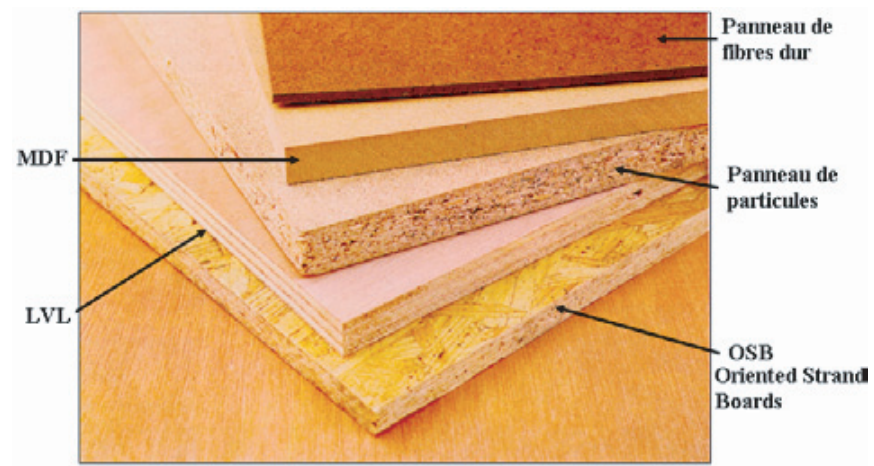

Fig. 1. Exemples de panneaux composites bois.

- Et ceux économiques (compenser la dégradation qualitative de la ressource forestière par la fabrication de matériaux d'ingénierie aux performances prévisibles).

Le travail entrepris a fait appel à deux techniques d'études des propriétés des composites, l'une mécanique, de simulation de fraisage (toupillage) et de mesure d'état de 
surface, et l'autre physique, d'analyse de la densité des panneaux.

Les composites testés sont :

Medium : $\quad$ panneaux fibres $16 \mathrm{~mm}$ (MDF 16)

Novoplac: $\quad$ panneaux particules $16 \mathrm{~mm}$ (16 1N P2)

Barapan : panneaux particules $16 \mathrm{~mm}$ (16 2N L2)

Novoplac: panneaux particules $16 \mathrm{~mm}$ code $\mathrm{P}$ (16 3N P2)

Novoplac: $\quad$ panneaux particules $14 \mathrm{~mm}$ (14 3C P2)

Triply OSB panneaux particules $\mathrm{CH} 10 \mathrm{~mm}$ code NP CH 10 NP)

L'essentiel des résultats nous a été donné par ces deux méthodes utilisées en parallèle, et nous avons calculé les corrélations entre les séries de données de l'une et l'autre méthode.

Les échantillons examinés ne peuvent qu'être de petites dimensions $\left(16 \mathrm{~mm}^{*} 30 \mathrm{~mm}^{*} 130 \mathrm{~mm}\right)$.

C'est pourquoi cette phase est appelée simulation d'usinage.

\section{Méthode et moyens}

\subsection{Simulation d'usinage}

Cette partie de l'étude a été faite sur le banc d'usinage (Zerizer $[1,2]$ ) mis au point dans le laboratoire d'usinage de I'E.N.S.T.I.B. (Université de Nancy). Ce dispositif, non seulement simule sur des échantillons de taille réduite des opérations de fraisage (toupillage), mais encore permet de mesurer et d'enregistrer les puissances et efforts provoqués par l'outil sur les échantillons testés (Fig. 2).

Ce dispositif de fraisage peut être réglé pour simuler diverses conditions : types d'outils, profondeur de passe, vitesse d'amenage, fréquence de rotation. . . et déterminer les meilleurs réglages à adopter.

Après chaque essai, le calculateur nous donne :

- La valeur de l'effort maxi de coupe suivant $Y$ et $Z$.

- La moyenne des efforts maxi suivant $Y$ et $Z$.

- Le temps de mesure.

- La valeur de la puissance électrique moyenne.

Pour les essais relatifs à la présente étude, nous avons défini différentes séries de relevés par rapport aux paramètres de coupe définis préalablement.

Le calcul des efforts de coupe se fait sur les pics mesurés lors de l'acquisition des données. Un pic correspond à l'effort maxi mesuré lors du passage d'une des dents de l'outil dans le matériau. La variation de l'effort subi par une dent de l'outil lors de son passage dans le matériau est présentée (Fig. 3).

Les échantillons (au total 54 éprouvettes ont été sélectionnées) devant servir aux essais ont été tirés des panneaux cités au préalable et usinés avec un outil carbure de diamètre $170 \mathrm{~mm}$ pour une fréquence de rotation de 6000 tr. $\min ^{-1}$ pour des combinaisons respectives de vitesse d'amenage $\left(17,20\right.$ et $\left.23 \mathrm{~m} . \mathrm{min}^{-1}\right)$ et profondeurs de passe $(2,2,5$ et $3 \mathrm{~mm})$.

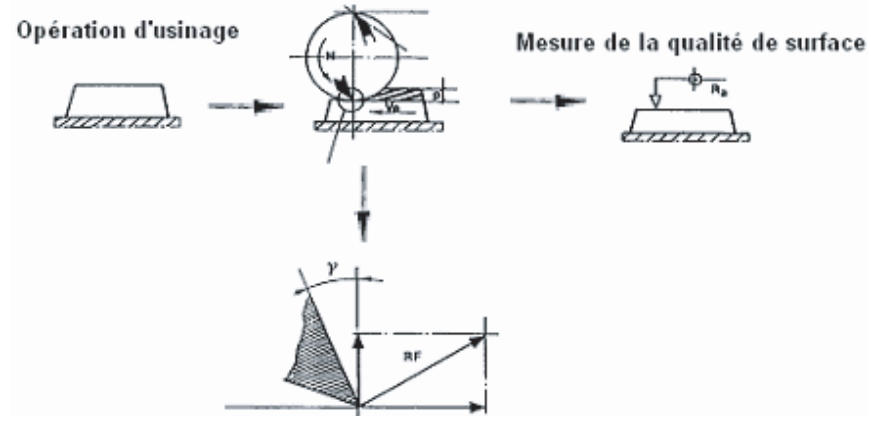

Fig. 2. Banc d'essai d'usinage.

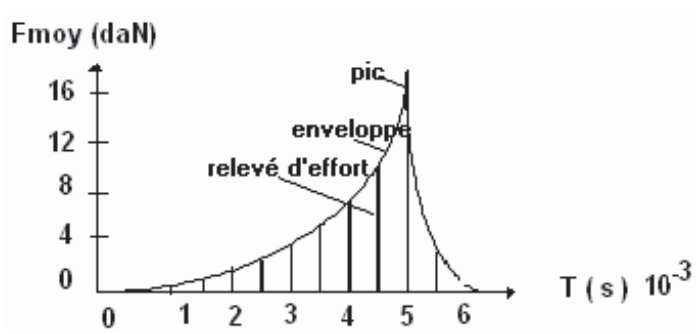

Fig. 3. Relevé d'un effort de coupe.

\subsection{Matériel de mesure}

\subsubsection{La platine de mesure}

Les efforts suivant les 3 directions seront mesurés à l'aide d'un dynamomètre de type Kistler 9257A dont le principe est basé sur les propriétés physiques des matériaux piézo-électriques (les surfaces de certains cristaux se chargent électriquement lorsque le cristal est soumis à des sollicitations mécaniques). La plateforme est conçue pour la mesure des forces selon trois composantes $(X, Y$ et $Z)$. Tous les canaux $X, Y, Z$ sont branchés respectivement en parallèle et la mesure est indépendante du point d'application de la force.

\subsubsection{Les amplificateurs}

Ces appareils ont pour but de convertir la charge électrique délivrée par la platine en une tension électrique proportionnelle en tenant compte de la sensibilité du capteur $(N / V)$ d'où le choix de la gamme de mesure.

\subsubsection{La carte de conversion PA300}

Au cours des mesures, les amplificateurs délivrent un signal analogique. Pour être traité puis enregistré, ce signal sera échantillonné à une fréquence égale à $35 \mathrm{KHz}$ sur chaque voie (Fig. 4).

Cette carte est pourvue d'un connecteur à 62 pôles pour être directement insérée dans un slot de microordinateur. 

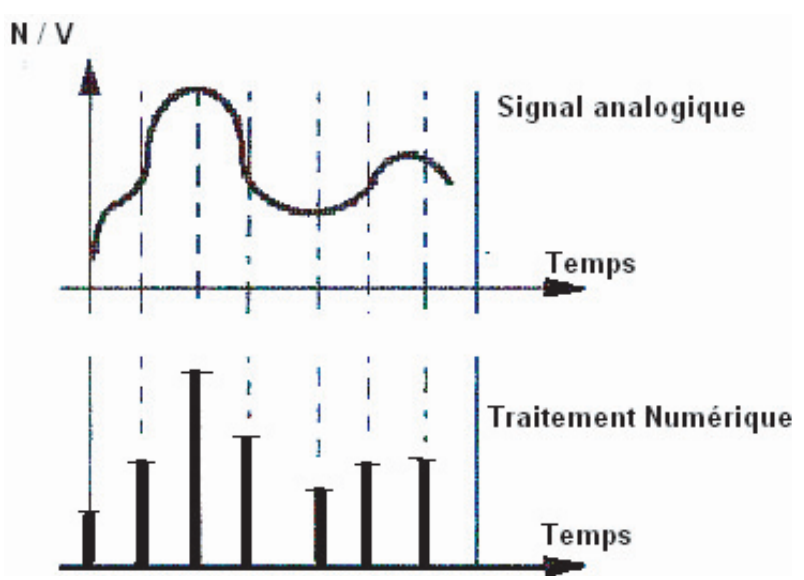

Fig. 4. Acquisition du signal.

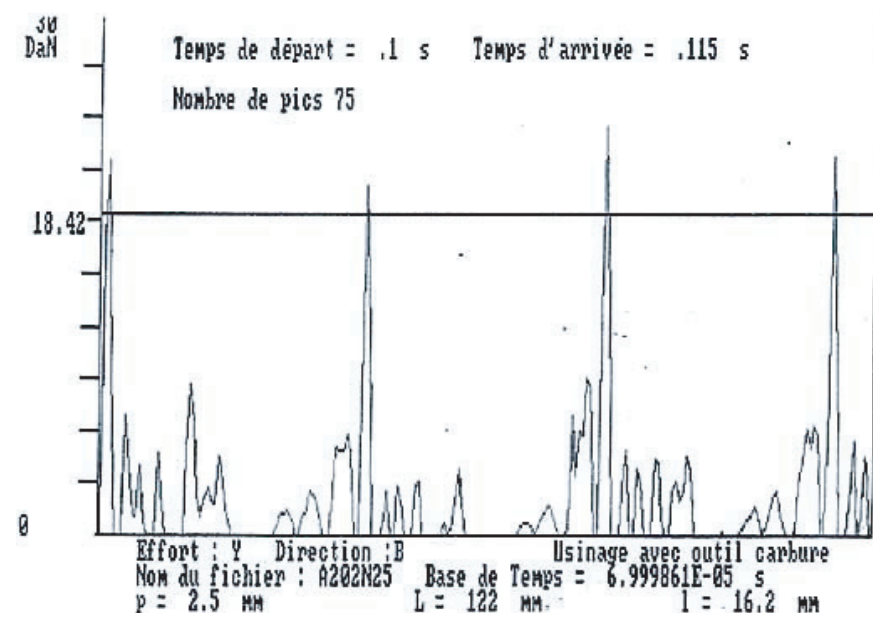

Fig. 5. Copie d'écran d'un effort de coupe relevé suivant $Y$ et dans la direction d'usinage $B$.

\subsubsection{Saisie des mesures}

La saisie de mesures a été possible suite à une écriture d'un programme facilitant l'introduction des données suivantes : gamme utilisée sur l'amplificateur de charge, profondeur de passe, longueur de la pièce à usiner, largeur de l'éprouvette (épaisseur des panneaux), type d'outil utilisé, fréquence de rotation de l'arbre, direction de coupe, voie de mesure ( $F y$ dans notre cas) opposée au mouvement d'avance de l'échantillon.

Après chaque essai d'usinabilité, la visualisation graphique nous donne la valeur de l'effort de coupe moyen, le temps de mesure, le nombre de pics (Fig. 5).

Suivant l'homogénéité du MDF les valeurs des pics sont plus ou moins dispersées. Quelques pics situés très haut peuvent faire augmenter l'effort moyen qui est calculé par le rapport de la somme des pics sur le nombre de pics (nombre de coups de dents) et l'effort maxi correspond au pic maxi. Pour la puissance électrique consommée au cours de l'usinage des éprouvettes, la prise d'information se fait directement sur les câbles d'alimentation électrique du moteur de broche par l'intermédiaire d'un wattmètre. Les mesures sont reportées sur table
Tableau 1. Valeurs expérimentales des densités et humidités.

\begin{tabular}{ccc}
\hline Désignation & Densité moyenne & Humidité moyenne \% \\
\hline 14 3C NF & 0,695 & 8,98 \\
16 3N P2 & 0,577 & 8,61 \\
16 1N P2 & 0,661 & 8,32 \\
CH 10 NP & 0709 & 8,00 \\
MDF 16 & 0,711 & 757 \\
16 N P2 & 0,716 & 8,27 \\
\hline
\end{tabular}

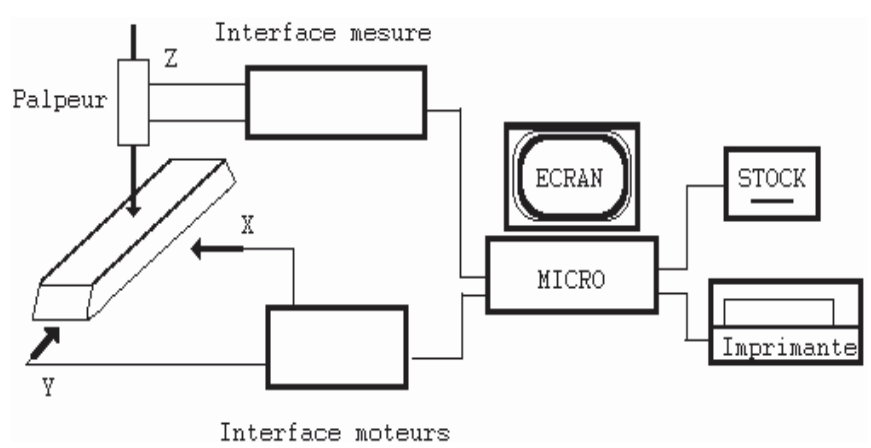

Fig. 6. Banc de mesure d'états de surface.

traçante (l'échelle est obtenue en mesurant la puissance à vide de la machine) ou calculées directement au moyen du calculateur.

\subsection{Mesure de la densité}

La densité est un facteur déterminant de l'aptitude du bois à l'effort de coupe et à l'usinage (Duong et Saison [3], Frantz [4], Movassaghi [5], Thomas [6]). Un calcul de densité et d'humidité a été effectué afin de connaître les caractéristiques physiques des panneaux composites bois au moment du relevé des efforts de coupe (Tab. 1).

La présence d'un profil de densité au sein des différents panneaux est un important paramètre d'hétérogénéité qui pourrait influencer les liaisons efforts de coupe, densité et états de surface.

\subsection{Mesure des états de surface}

\subsubsection{Matériel de mesure}

L'installation du banc de mesure d'état de surface (Lambert [7]) est composée de (Fig. 6) :

- un micro-ordinateur

- une table à mouvements croisés $X Y$

- un rugosimètre Perthometer PRK

- électronique de commande

- support de table et rugosimètre.

\subsubsection{Procédure expérimentale}

Le contrôle et la qualification de l'état de surface deviennent de plus en plus un critère d'usinabilité, grâce 


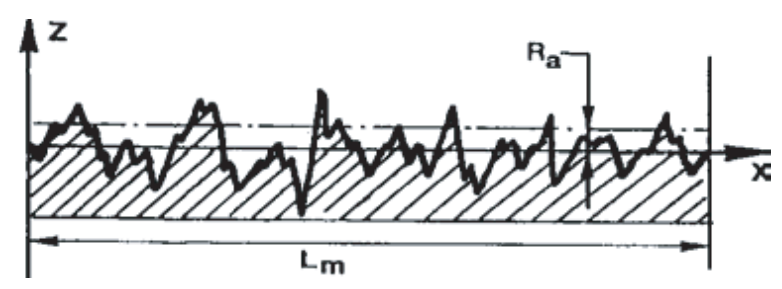

Fig. 7. Description de la surface à travers le critère $R_{\mathrm{a}}$.

notamment, aux progrès technologiques réalisés dans le domaine de la conception de nouvelles machines aux techniques avancées d'usinage. Selon Weil [8], une pièce ne correspond jamais exactement au dessin qui a servi à définir la forme géométrique même si elle reste à l'intérieur des tolérances géométriques imposées, elle possède des défauts dont il convient de limiter les grandeurs. En ce qui concerne notre démarche, le but principal est d'établir une relation entre les paramètres de coupe retenus et les états de surface issus de la profilométrie avec le principe suivant. Les échantillons sont fixés sur la table $X Y$ mobile, et les déplacements mesurés par un palpeur dont l'aiguille exploratrice (rayon de pointe de $10 \mu \mathrm{m}$ ) parcourt une ligne droite sur la surface du chant du panneau en suivant les irrégularités de la surface usinée. Les déplacements sont transmis à un capteur et les variations détectées par le stylet (dépressions et pics) sont transcrites, et agrandies sur un graphique. La table $X Y$ représente les deux déplacements : l'un dans le sens du relevé du profil et l'autre dans un sens perpendiculaire au premier. Le choix des directions de mesure est prépondérant et les résultats issus de l'étude des profils ne peuvent donner qu'une vision partielle de l'état réel de la surface.

\subsubsection{Critères classiques de rugosité}

Il est à noter que le banc de mesure d'états de surface délivre généralement les $R_{\mathrm{a}}, R_{\mathrm{p}}, R_{\mathrm{z}}, R_{\max }, R_{\mathrm{t}}$ ce qui présente l'avantage de donner sous la forme d'un nombre une information précise sur le profil étudié.

Parmi les critères pouvant être utilisés pour l'analyse de nos éprouvettes, nous avons retenu l'écart moyen arithmétique $R_{\mathrm{a}}$, plus couramment utilisé dans la littérature (Fig. 7) :

$R_{\mathrm{a}}$ écart moyen arithmétique de la rugosité par rapport à la ligne moyenne ${ }^{1}$

$$
R_{\mathrm{a}}=\frac{1}{L_{\mathrm{m}}} \int_{0}^{L_{\mathrm{m}}}\left|Z-R_{\mathrm{p}}\right| \mathrm{d} x
$$

\footnotetext{
${ }^{1}$ La ligne moyenne représente l'ondulation et la forme, elle coupe le profil pour obtenir une répartition symétrique des surfaces des irrégularités supérieures et inférieures.
}

$R_{\mathrm{p}}$ : capacité d'aplanissement maximale, hauteur évaluée par rapport à la ligne moyenne du pic le plus haut rencontré sur la longueur totale $l_{\mathrm{m}}$

$$
R_{\mathrm{p}}=\frac{1}{L_{\mathrm{m}}} \int_{0}^{L_{\mathrm{m}}} Z \mathrm{~d} x
$$

\section{Résultats}

Les résultats obtenus étaient suffisamment significatifs pour que l'on poursuive ce type d'étude à l'ensemble des panneaux. Nous avons recherché les corrélations existantes entre les efforts de coupe, les états de surfaces et les variables d'action : paramètres de coupe et densité. Nous présentons dans ce qui suit, les relevés des différentes courbes lesquelles seront suivies d'un commentaire expliquant les relations trouvées.

\section{Commentaires}

La faible corrélation entre l'effort de coupe moyen et les facteurs étudiés : épaisseur moyenne du copeau et densité (Figs. 8, 11 et 12) est notamment due à la prépondérance de l'hétérogénéité des panneaux par rapport à la constitution et densité des panneaux : taille des particules et séparation involontaire résultant des vibrations pendant le transport du «gâteau » lors de la fabrication du panneau. Le profil de densité n'étant pas symétrique sur toute l'épaisseur du panneau (Fig. 13), cela entraîne au cours de l'usinage des efforts de coupe variables, ce qui explique en partie les faibles corrélations résultantes de l'étude.

Par ailleurs, il faut noter que l'influence de l'effort par rapport à l'épaisseur moyenne du copeau est due à la faible gamme des profondeurs de passe utilisées. C'est pourquoi, il serait intéressant pour la suite de ce travail, d'étendre les valeurs en prenant des passes différentes afin de mieux expliquer ces paramètres. Pour ce qui est de la puissance de coupe, nous dirons pendant le travail d'enlèvement du copeau, que la puissance consommée devient beaucoup plus importante au fur et à mesure de la pénétration de l'outil dans la matière. Les variations des résultats s'expliquent par les vibrations induites au moment de la prise du copeau auxquelles s'ajoutent celles engendrées par les mécanismes de la machine (Fig. 2) et l'hétérogénéité du matériau.

\section{Conclusion}

En conclusion de cette étude, et malgré les difficultés nombreuses qui sont apparues au cours de la phase expérimentale, et qui nous ont empêchés de procéder à toutes les mesures prévues, il semble bien que les résultats obtenus soient très encourageants.

Les variations de la densité ainsi que des paramètres de coupe que sont les vitesses d'amenage, la profondeur de 


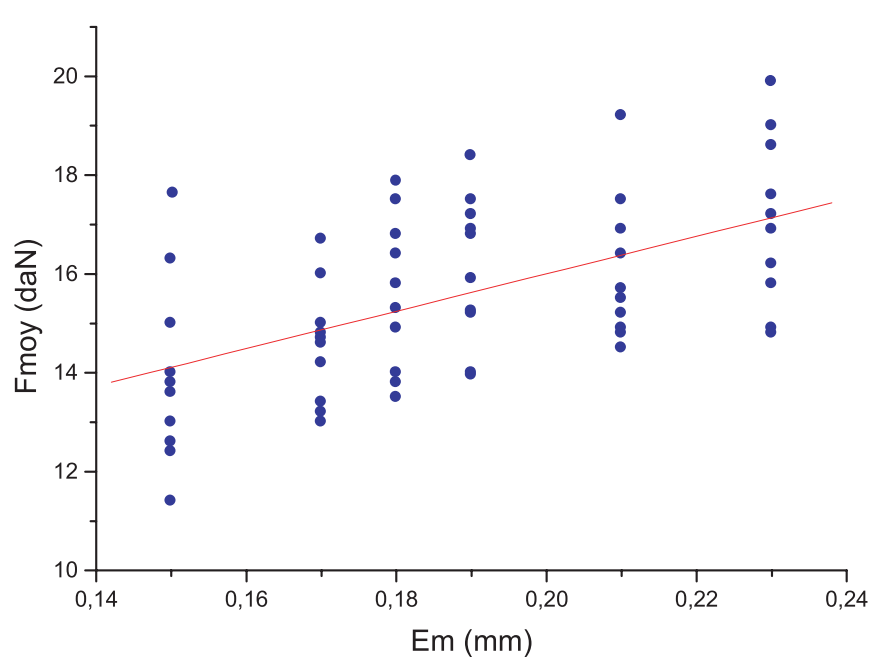

Fig. 8. Influence de $E_{\mathrm{m}}$ sur $F_{\text {moy }}\left(F_{\text {moy }}=6,99+46,94 E_{\mathrm{m}}\right.$; $\left.R=0,63^{* *}\right)$.

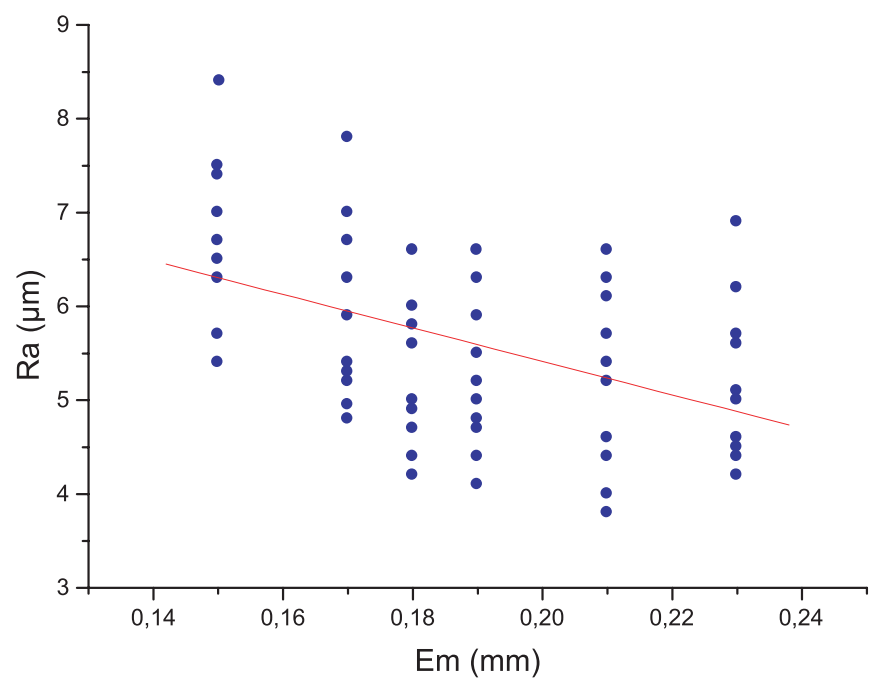

Fig. 9. Influence de $E_{\mathrm{m}}$ sur $R_{\mathrm{a}}\left(R_{\mathrm{a}}=9,11-18,19 E_{\mathrm{m}} ; R=\right.$ $\left.-0,48^{* *}\right)$

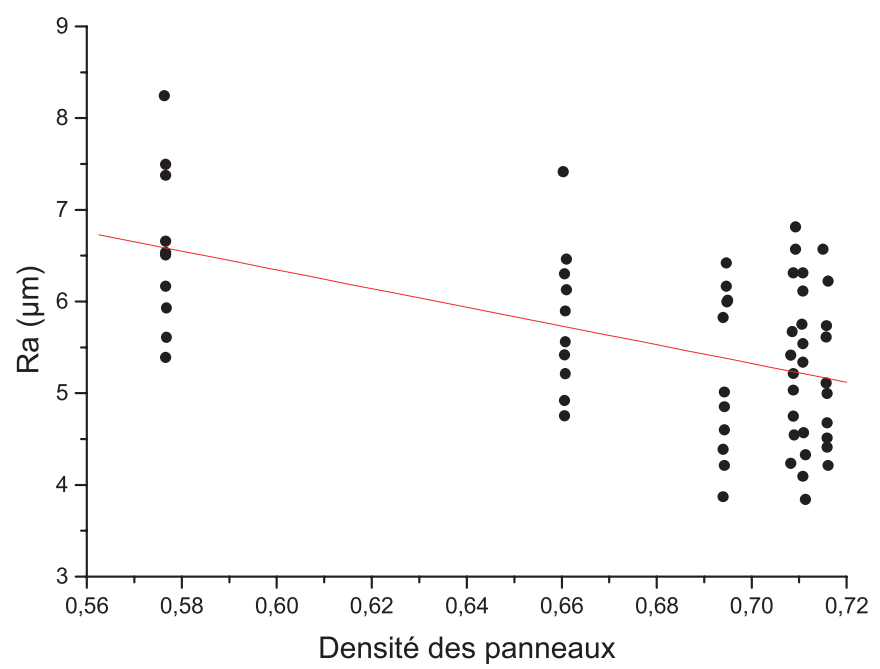

Fig. 10. Influence de la densité sur $R_{\mathrm{a}}\left(R_{\mathrm{a}}=13,50-11,78 D\right.$; $\left.R=-0,52^{* *}\right)$.

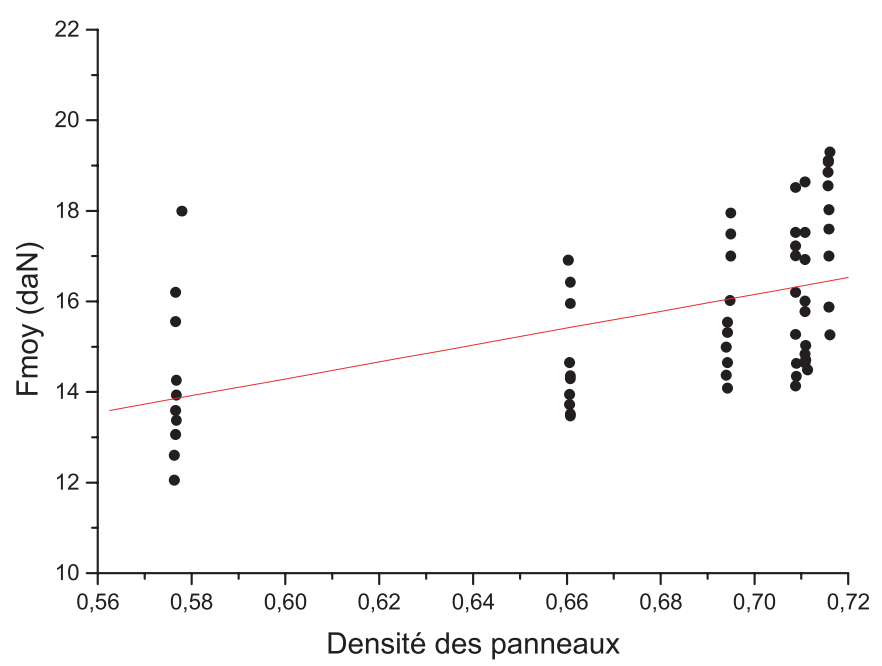

Fig. 11. Influence de la densité sur $F_{\text {moy }}\left(F_{\text {moy }}=1,04+\right.$ $\left.21,85 D ; R=0,56^{* *}\right)$.

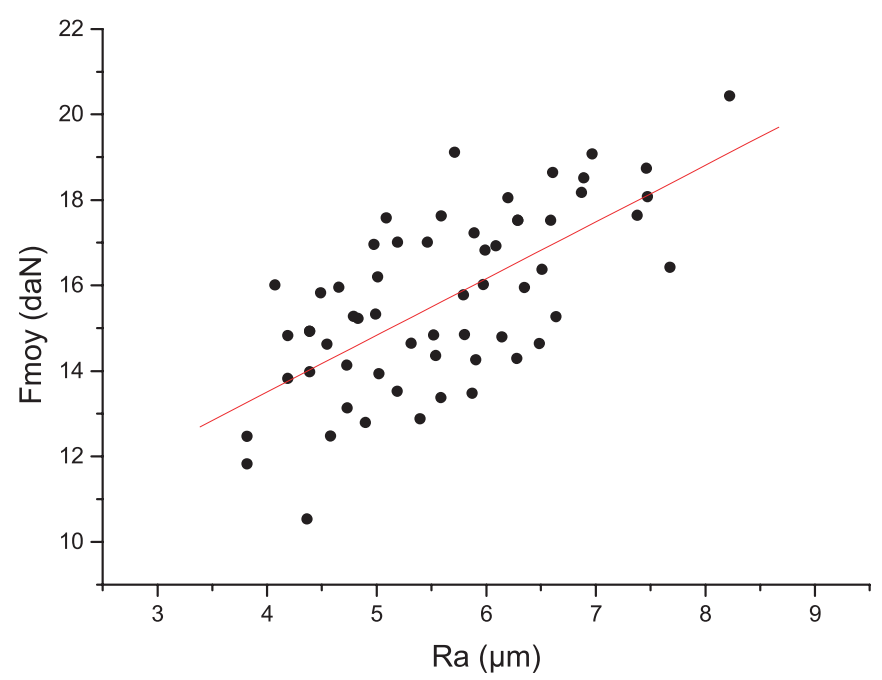

Fig. 12. Influence de l'écart arithmétique moyen sur l'effort de coupe $\left(F_{\text {moy }}=7,87+1,27 R_{\mathrm{a}} ; R=0,56^{* *}\right)$.

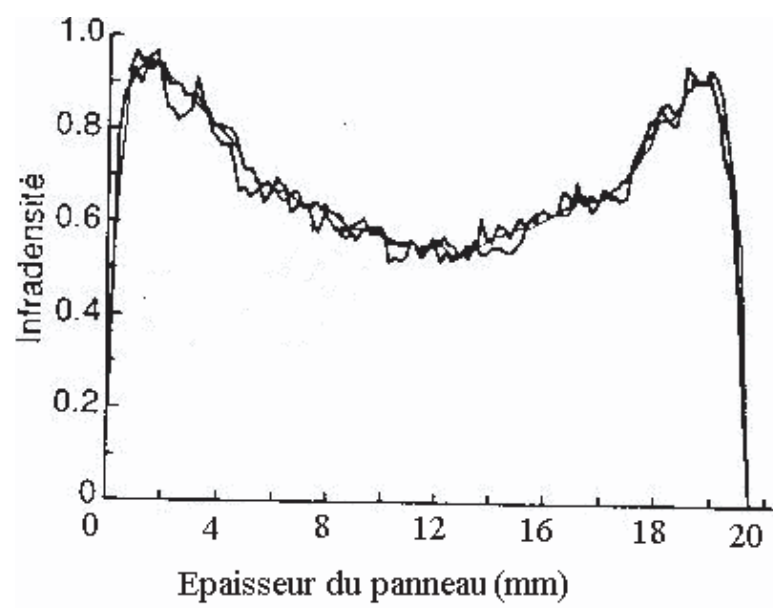

Fig. 13. Profil de densité d'un panneau de particules. 
passe et la fréquence de rotation représentés à travers $E_{\mathrm{m}}$ (épaisseur moyenne du copeau) jouent un rôle explicatif important pour ce qui est de la qualité d'usinage.

À la suite de cette procédure, on peut insister sur la nécessité d'utiliser une matière première la moins hétérogène possible, donc de favoriser les pratiques permettant d'atteindre ce but. La densité a une action plus marquée sur les efforts et les panneaux en matière d'état de surface restent dépendant de leur composition, de leur équilibre hygroscopique et de leur stabilité, c'est pourquoi il aurait été intéressant de connaître les conditions initiales de fabrication facilitant au mieux les études spécifiques sur l'usinabilité. Ces études constituent un point de départ nécessaire pour l'identification des meilleurs paramètres de coupe et de la détermination de la zone de bon fonctionnement (concept du couple outil matière) (Aguilera [9], AFNOR [10]).

\section{Références}

[1] A. Zerizer, Étude des efforts de coupe et de l'état de surface en toupillage, DEA Sciences du bois LACN Université de Nancy I, 1988

[2] A. Zerizer, Contribution à l'étude de l'usinabilité du MDF (Medium Density Fiberboard), thèse de Doctorat de l'université de Nancy I en sciences du bois, 177 p., 1991

[3] L. Duong Vinh, R. Saison, Travail mécanique du bois, Techniques de l'ingénieur, B7, 1800-1808

[4] F.C. Frantz, An analysis of the wood cutting process Ph.D. thesis, University of Michigan Press Ann Arbor, 1958

[5] E. Movassaghi, Influence des paramètres microdensitométriques du bois sur les efforts de coupe et la qualité des placages de Douglas et Châtaignier obtenus par déroulage, thèse de Doctorat Ingénieur USTL Montpellier, 1985

[6] C.E. Thomas, Étude bibliographique des facteurs influençant les efforts de coupe dans l'usinage du matériau bois, DEA Sciences du bois LACN Université de Nancy I, 1984

[7] P. Lambert, Réalisation d'un système de mesure d'état de surface pour le matériau bois DEA Génie électrique LACN Université de Nancy I, 1985

[8] R. Weil, Techniques d'usinage, Éditions Dunod, 1971

[9] A. Aguilera, Optimisation des conditions de coupe pour l'usinage du bois. Application de la méthode du couple Outil Matière au défonçage des panneaux de fibres de densité moyenne, $\mathrm{Ph}$.D. thesis in wood science, université de Nancy I, France, 2000

[10] AFNOR, Domaine de fonctionnement des outils coupants, Couple Outil Matière. Partie 6 : mode d'obtention du Couple Outil Matière en fraisage. Indice de classement NFE66-520-6, 1995

Retrouvez nos articles sur le site : www.edpsciences.org/meca 